\title{
Prediction of Postoperative Cerebral Infarction after Cardiovascular Surgery Using Quantitative Measurement of Cerebral Blood Flow with Brain Single-Photon Emission Computed Tomography
}

\author{
Hiroshi Tomoeda, MD, PhD, ${ }^{1}$ Keita Mikasa, MD, ${ }^{2}$ Shingo Chihara, MD, PhD, ${ }^{3}$ \\ Kentaro Sawada, MD, PhD, ${ }^{4}$ and Hiroyuki Tanaka, MD, PhD ${ }^{5}$
}

Prediction of postoperative cerebral infarction after cardiovascular surgery is difficult. The present study investigated whether quantitative evaluation of preoperative cerebral blood flow used in the Japanese EC-IC Bypass Trial (JET) study is useful for the prediction of postoperative cerebral infarction after cardiovascular surgery. First, patients were divided into two groups based on preoperative cerebral blood flow. In an evaluation using preoperative imaging, patients with good or mildly decreased preoperative cerebral blood flow, divided into clinical stage I or II by quantitative evaluation showed no postoperative cerebral infarction. However, $24 \%$ of patients with poor cerebral blood flow who were categorized as clinical stage II, experienced postoperative cerebral infarction. The incidence rate was not statistically significantly different when the groups were compared. Second, patients were divided into two groups based on the anatomical area of the brain affected corresponding to clinical stage II. Patients with a $10 \%$ and

${ }^{1}$ Department of Cardiovascular Surgery, Chikugo City Hospital, Chikugo, Fukuoka, Japan

${ }^{2}$ Department of Surgery, Iizuka City Hospital, Iizuka, Fukuoka, Japan

${ }^{3}$ Department of Cardiovascular Surgery, Yokokura Hospital, Miyama, Fukuoka, Japan

${ }^{4}$ Department of Surgery, Saiseikai Futsukaichi Hospital, Chikushino, Fukuoka, Japan

${ }^{5}$ Department of Cardiovascular Surgery, Kurume University School of Medicine, Kurume, Fukuoka, Japan

Received: September 27, 2018; Accepted: September 28, 2018 Corresponding author: Hiroshi Tomoeda, MD, PhD. Department of Cardiovascular Surgery, Chikugo City Hospital, 917-1 Izumi, Chikugo, Fukuoka 833-0041, Japan

Tel: +81-942-53-7511, Fax: +81-942-53-7515

E-mail: tomoeda.hiroshi@gmail.com

This is a translation of J Jpn Coll Angiol 2017; 57: 125-133.

(cc) BY-NC-SA (92018 The Editorial Committee of Annals of Vascular Diseases. This article is distributed under the terms of the Creative Commons Attribution License, which permits use, distribution, and reproduction in any medium, provided the credit of the original work, a link to the license, and indication of any change are properly given, and the original work is not used for commercial purposes. Remixed or transformed contributions must be distributed under the same license as the original. greater brain involvement had a significantly higher incidence of postoperative cerebral infarction (38\%) compared to others $(0 \%, p<0.01)$. This method may be useful for the prediction of postoperative cerebral infarction after cardiovascular surgery, but a further prospective study is needed. (This is a translation of J Jpn Coll Angiol 2017; 57: 125-133.)

Keywords: postoperative cerebral infarction, cardiovascular surgery, JET-study, brain SPECT

\section{Introduction}

Cerebral infarction following cardiovascular surgery reduces patient's quality of life (QOL), increases mortality, prolongs hospital stay, and increases medical costs. Consequently, stroke is a primary factor that determines whether surgery should be performed. With the recent increase in age of patients receiving cardiovascular surgery, postoperative neuropathy complications pose a problem. For reducing the incidence of these complications, various studies on surgical procedures and the use of intraoperative cerebral blood flow monitoring have been conducted. However, it is difficult to evaluate the risk of stroke purely on the basis of the morphological findings of blood vessels using preoperative computed tomography (CT) and magnetic resonance imaging (MRI), and there are no established evaluation methods. The JET study ${ }^{1)}$ was the first worldwide study to obtain evidence of the clinical effectiveness of superficial temporal artery (STA)-middle cerebral artery (MCA) bypass in the field of brain surgery, and we examined whether the cerebral blood flow measurement used in that study can serve as a useful measure for predicting the onset of postoperative stroke.

\section{Subjects and Methods}

\section{Methods}

A total of 535 patients had undergone elective cardiovascular surgery at the Department of Surgery of Kurume University Hospital between June 2009 and February 
2012. We excluded patients who underwent direct manipulations of the cervical branch, such as selective cerebral perfusion, and patients who were determined to require carotid endarterectomy (CEA) on close examination preoperatively and thus underwent CEA for carotid stenosis. Lastly, we included the remaining 33 patients who exhibited $\geq 50 \%$ stenosis on the left or right or bilateral carotid arteries on carotid ultrasonography using a linear probe [according to the method in the European Carotid Surgery Trial (ECST)] performed for preoperative examination, and who provided consent to undergo cerebral blood flow measurement. Carotid ultrasonography was performed using prosound 2 (Aloka) with a linear probe set to $10 \mathrm{MHz}$.

Preoperative cerebral blood flow was measured in these 33 patients using the method described in the abovementioned Japanese extracranial-intracranial Bypass Trial (JET) study.

Unlike brain single photon emission computed tomography (SPECT), which is frequently used, this method of measuring cerebral blood flow levels involved obtaining cerebral blood flow measurements on the basis of brain SPECT values of different locations at rest and with a venous infusion loading of $1,000 \mathrm{mg}$ of acetazolamide. On combining the blood radiation level measurements from arterial blood samples while obtaining SPECT measurements, the cerebral blood flow level can be measured per minute for different locations of the brain, and the rate of increase in cerebral blood flow upon acetazolamide loading can be measured. The rate of cerebral blood flow measured and the rate of increase in cerebral blood flow (cerebral blood flow reserve) on acetazolamide loading were classified into stages $0-$ II according to the severity as per the classification of hemodynamic cerebral ischemia. ${ }^{2}$ This classification was rendered into three-dimensional (3D) images of the brain that indicated the risk of ischemia for each location of the brain.

Stage 0 cerebral blood flow is shown in pale green on $3 \mathrm{D}$ images of the brain in patients with normal-to-reduced cerebral blood flow at rest, with a $\geq 30 \%$ increase in cerebral blood flow displayed upon acetazolamide loading; stage 0 is the most favorable cerebral blood flow in the staging classification. Stage I, which indicates cerebral blood flow between stages 0 and II, is shown in dark green on $3 \mathrm{D}$ images in patients with normal cerebral blood flow at rest, and it displays a $10 \%-30 \%$ increase in cerebral blood flow upon acetazolamide loading. Stage II indicates locations with the highest risk of ischemia in the classification and is shown in orange on 3D images in patients with a cerebral blood flow at rest of at least $20 \%$ less than the normal mean; stage II displays a $<10 \%$ increase in cerebral blood flow upon acetazolamide loading.

Precautions are exercised for patients with stage II cerebral blood flow in whom cerebral blood flow and blood flow reserve are judged to be poor preoperatively. If possible, in coronary artery bypass surgery, we performed off-pump bypass surgery, wherein postoperative stroke onset is considered unlikely, and for operations using a cardiopulmonary bypass $(\mathrm{CPB})$ pump, we adopted measures that increase the CPB flow rate during extracorporeal circulation.

The present study was conducted with the approval of the ethical review board of the Kurume University School of Medicine, and written consent was obtained from each study subject after a thorough explanation of the study.

\section{Subjects}

The sample population consisted of 33 patients aged 54-84 (68.7 \pm 8.54$)$ years, including 23 males and 10 females.

In the 33 subjects, the mean rate of stenosis on carotid ultrasonography (in the event of bilateral stenosis, the more severe side) was $70.6 \% \pm 24.1 \%$ on the basis of the linear probe method (ECST method) and $77.5 \% \pm 17.7 \%$ based on the area method.

Tables 1 and 2 present the preoperative patient background data according to each stage of cerebral blood flow classification.

Table 1 Clinical characteristics and operative procedure at each clinical stage according to quantitative cerebral blood flow

\begin{tabular}{|c|c|c|c|c|c|}
\hline & Number of patients & Age & $\operatorname{Sex}(M / W)$ & Operative proc & \\
\hline \multirow[t]{3}{*}{ Stage 0} & 5 & $71.6 \pm 10.1$ & $3 / 2$ & Valvular & 2 \\
\hline & & & & CABG (on pump) & 1 \\
\hline & & & & OPCAB & 2 \\
\hline \multirow[t]{3}{*}{ Stage I } & 4 & $70.2 \pm 6.2$ & $2 / 2$ & Valvular & 2 \\
\hline & & & & OPCAB & 1 \\
\hline & & & & OPCAB 1 & 1 \\
\hline \multirow[t]{4}{*}{ Stage II } & 24 & $67.8 \pm 8.7$ & $18 / 6$ & Valvular & 5 \\
\hline & & & & CABG (on pump) & 5 \\
\hline & & & & OPCAB & 10 \\
\hline & & & & Others & 4 \\
\hline
\end{tabular}


Table 2 Clinical characteristics at each clinical stage according to quantitative cerebral blood flow

\begin{tabular}{|c|c|c|c|c|c|c|}
\hline & Number of patients & preOP T-LOC & preOP diz & $\%$ sten of cerebral L & $\%$ sten of cerebral A & $\%$ severe stenosis of ICA \\
\hline \multirow[t]{4}{*}{ Stage 0} & 5 & 0 & 0 & $74.3 \pm 17.4$ & $81.6 \pm 14.8$ & BSCA $60 \%$ \\
\hline & & & & & & Basilar artery $0 \%$ \\
\hline & & & & & & ACA $0 \%$ \\
\hline & & & & & & MCA $60 \%$ \\
\hline \multirow[t]{4}{*}{ Stage I } & 4 & 0 & 0 & $64.9 \pm 11.8$ & $69.0 \pm 9.5$ & BSCA $50 \%$ \\
\hline & & & & & & Basilar artery $0 \%$ \\
\hline & & & & & & ACA $0 \%$ \\
\hline & & & & & & MCA $60 \%$ \\
\hline \multirow[t]{5}{*}{ Stage II } & 24 & 4 & 2 & $69.0 \pm 19.9$ & $77.6 \pm 19.3$ & BSCA $43 \%$ \\
\hline & & & & & & Basilar artery $13 \%$ \\
\hline & & & & & & ACA $22 \%$ \\
\hline & & & & & & MCA $39 \%$ \\
\hline & & & & & & PCA 4\% \\
\hline
\end{tabular}

preOP T-LOC: preoperative transient loss of consciousness; preOP diz: preoperative dizziness; \% sten of cerebral L: percentage of ECST stenosis (more severe side); \% sten of cerebral A: percentage of stenotic area of cerebral artery (more severe side); \% severe stenosis of ICA: percentage of severe stenosis of intracranial artery (more severe side); BSCA: the base of the skull at the carotid artery; ACA: anterior cerebral artery; MCA: middle cerebral artery; PCA: posterior cerebral artery

The patients underwent MRI of the head preoperatively and early postoperatively (within six days following surgery). In the early stage, image diagnosis was abandoned for four patients in whom early postoperative MRI could not be performed for reasons including the inability to remove the extracorporeal pacing wire within six days following surgery. In the patients in whom the pacing wire could not be removed after that, postoperative head CT was performed, and the presence or absence of perioperative stroke was determined by comparing pre- and postoperative CT images. MRI of the head was performed using a Signa HDxt1.5T from GE Healthcare Japan, and CT of the head was performed using a LEMAGE SX/E from GE Healthcare Japan. To determine the rate of severe stenosis of intracranial arteries as shown in Table 2 , severe stenosis was diagnosed when patients in each cerebral blood flow classification stage had lesions with approximately $\geq 75 \%$ stenosis in each location of the intracranial arteries on MRI imaging. Furthermore, on the plain CT of the chest performed for close examination preoperatively, none of the 33 patients exhibited clear abnormality of the ascending aorta.

\section{Results}

For each patient, the cerebral blood flow reserve on the basis of the cerebral blood flow measurement was classified into stages for each brain location. These measurements were rendered into a 3D model of the brain. In some patients, different brain locations displayed different stages of cerebral blood flow. First, on the basis of the cerebral blood flow reserve classifications of each patient, we conducted an analysis wherein the worst stage of classification was defined as that patient's cerebral blood flow stage. Nine of the 33 patients were classified as stage 0 or I, and 24 patients were classified as stage II. We conducted a statistical examination for determining whether there was a significant difference in the degree of stenosis on a preoperative carotid ultrasonography between nine patients with stage 0 or I and 24 patients with stage II. The degree of carotid artery stenosis on the side with severe stenosis in the nine patients with stage 0 or I was $70.3 \% \pm 14.9 \%$ on the basis of the linear probe (ECST method) and $76.9 \% \pm 13.9 \%$ on the basis of the area method. However, for the 24 patients with stage II, the degree of stenosis on the side with severe stenosis was $69.0 \% \pm 19.9 \%$ on the basis of the linear probe (ECST method) and $77.6 \% \pm 19.3 \%$ on the basis of the area method. The degree of stenosis in these patients was examined using a Wilcoxon rank sum test because of the absence of a normal distribution on the Shapiro-Wilk test. The results revealed $\mathrm{p}=0.49$ and $\mathrm{p}=0.63$, respectively, and no significant difference was observed. The analyses were performed using JMP 10 (Version 10.01) software (SAS Inc.).

Next, in the 24 patients with stage II cerebral blood flow based on scintigraphy, we examined whether the sides with severe carotid stenosis on carotid ultrasound using a linear probe (ECST method) and with severe hemisphere due to cerebral ischemia based on cerebral blood flow scintigraphy (the hemisphere with the largest portion of the surface area classified as stage II) tended to be statistically consistent. Furthermore, when the left-right difference in the rate of carotid artery stenosis was $<10 \%$, it was considered bilateral stenosis, and when the left-right difference in the proportion $(\%)$ of the surface area of the 
hemisphere classified as stage II based on cerebral blood flow scintigraphy was $<10 \%$, it was considered bilateral ischemia. The results revealed that out of the 24 patients with stage II, bilateral stenosis on carotid ultrasonography and bilateral ischemia on cerebral blood flow scintigraphy were observed in four patients, right predominance on ultrasonography and bilateral ischemia on scintigraphy were observed in six patients, left predominance on ultrasonography and bilateral ischemia on scintigraphy were observed in six patients, and other results were observed in eight patients. In total, $58 \%$ of the patients showed bilateral ischemia on scintigraphy, and Fisher's test using the EZR software (Version 1.32) ${ }^{3)}$ revealed no constant statistical tendency in unilateral stenosis and bilateral stenosis based on ultrasonography or in unilateral ischemia and bilateral ischemia based on cerebral blood flow scintigraphy $(\mathrm{p}=0.48)$.

Furthermore, we examined the rate of postoperative stroke onset for each ischemia stage classification. Among the 33 patients overall, nine patients were stage 0 or stage $\mathrm{I}$, and none of these nine patients showed neurologic symptoms suggestive of postoperative stroke. Among these nine patients, imaging was performed for early postoperative stroke examination in eight patients (MRI in six patients and CT in two patients), which revealed no perioperative stroke.

Next, we examined stage II patients with cerebral ischemia. Among the 33 patients, 24 patients were stage II, and one patient exhibited postoperative stroke accompanied with neurological symptoms (case 1 described below). The rate of postoperative stroke onset identified according to postoperative neurological symptoms was $4 \%$. Postoperative imaging was performed in 21 of the 24 patients to determine the presence of stroke (MRI in 20 patients and CT in 1 patient), and stroke was observed on the diagnostic imaging of five patients including case 1 . The rate of stroke onset according to image diagnosis was $24 \%$. In particular, examination of the patients who underwent off-pump bypass surgery only reveal that perioperative stroke was observed on diagnostic imaging in three out of the 10 patients with stage II (nine patients underwent postoperative image diagnosis); the rate of postoperative stroke onset according to such diagnostic imaging was high $(33 \%)$.

We tested whether there was a statistically significant difference in the rate of postoperative stroke onset between the eight patients with stage 0 or I and the $21 \mathrm{pa}$ tients with stage II who underwent imaging for postoperative stroke diagnosis. For the statistical analysis, Fisher's test was performed; however, no significant difference was observed $(\mathrm{p}=0.28)$ (analysis using EZR). ${ }^{3)}$

To investigate in further detail, we tested whether there was a significant difference in the rate of postoperative stroke onset between patients in whom stage II cerebral blood flow accounted for $\geq 10 \%$ of the surface area of the left hemisphere, right hemisphere, or bilaterally [the mean proportion of the location area showing stage II of the anterior cerebral artery (ACA) region, middle cerebral artery region, and posterior cerebral artery (PCA) region, respectively], and patients with stage II cerebral blood flow in $<10 \%$ of the bilateral hemisphere surface area.

Postoperative stroke did not occur in any of the 16 patients with stage 0 or I who underwent postoperative diagnostic imaging $(\mathrm{n}=8)$ or who displayed stage II in parts of the brain $(n=8)$; however, stage II cerebral blood flow occurred in $<10 \%$ of the surface area in the bilateral hemispheres. In the 13 patients who underwent postoperative diagnostic imaging, stage II cerebral blood flow occurred in $\geq 10 \%$ of the area of the left hemisphere, right hemisphere, or bilateral hemispheres on preoperative cerebral blood flow scintigraphy; of these 13 patients, postoperative stroke was observed in five patients, and the rate of postoperative stroke onset was $38 \%$. The results of Fisher's test using the EZR method ${ }^{3)}$ revealed that compared with the group with stage II cerebral blood flow in $<10 \%$ of the left hemisphere, right hemisphere, or bilateral hemispheres, the group with stage II cerebral blood flow in $\geq 10 \%$ of those areas was statistically significantly prone to stroke $(p=0.01)$. We also examined whether there was a statistically significant difference in the degree of carotid artery stenosis (linear probe and area method on the severe side) between the group with stage II cerebral blood flow in $\geq 10 \%$ of the area and the group with stage II cerebral blood flow in $<10 \%$; however, the t-test used in this study revealed no statistically significant difference with both the linear probe and area methods $(p=0.93$ and $\mathrm{p}=0.32$, respectively, using the EZR method ${ }^{3)}$ ).

Table 3 presents the patient backgrounds of the five patients with postoperative stroke onset determined by neurological symptoms and diagnostic imaging. Cerebral blood flow scintigraphy images and postoperative stroke images of each patient are presented (cerebral blood flow scintigraphy images: Figs. $1 \mathrm{a}-\mathbf{5}$ a, postoperative stroke images: Figs. $1 b-5 b)$.

In many of the patients who presented with stroke on the images above, the location of stroke onset was the region classified as stage II with poor cerebral blood flow on the cerebral blood flow scintigraphy, which is called the watershed zone. This location corresponds with the boundary of the area that supplies blood flow to each cerebral artery, which is believed to be prone to the poorest cerebral blood flow. Furthermore, in cases 2 and 3 mentioned above, as expected, preoperative scintigraphy revealed small infarctions in two locations, including one site each in area considered to be a watershed zone in locations classified as stage II. 
Table 3 Characteristics and important factors of the patients with postoperative cerebral infarction

\begin{tabular}{|c|c|c|c|c|c|c|c|c|}
\hline Case & Age/Sex & Symp./anomaly P.O. & Diagnosis & OP.procedure & $\begin{array}{l}\text { Clamp of Asc. } \\
\text { Ao/SVG empl. }\end{array}$ & $\begin{array}{c}\text { Most sten. } \\
\text { lesion/\%sten. }\end{array}$ & $\begin{array}{c}\text { Postoperative } \\
\text { AF }\end{array}$ & $\begin{array}{l}\text { Clin. stage/ } \\
\text { Postop. MRI }\end{array}$ \\
\hline 1 & 61/Male & dyspnea & CAD 3VD & $\mathrm{OPCAB} \times 3$ & No/Yes & Lt ICA/50\% & No & Fig. 1a/Fig. 1b \\
\hline 2 & 65/Male & vertigo & ASO & Ao-bif Bypass & No/No & Rt CCA $/ 79 \%$ & No & Fig. $2 a$ /Fig. $2 b$ \\
\hline 3 & 57/Male & angina and dyspnea & $\begin{array}{c}\text { CAD } \\
\text { LMT+1VD }\end{array}$ & OPCAB× 2 & No/No & Lt CCA $/ 50 \%$ & No & Fig. 3a/Fig. 3b \\
\hline 4 & 72/Male & dyspnea and leg edema & $\begin{array}{c}\text { CAD } \\
\text { LMT+1VD }\end{array}$ & $\mathrm{OPCAB} \times 4$ & No/Yes & Rt ICA/100\% & No & Fig. $4 \mathrm{a} /$ Fig. $4 \mathrm{~b}$ \\
\hline 5 & 69/Male & abnormal electrocardiogram & $\begin{array}{c}\text { CAD } \\
L M T+3 V D\end{array}$ & $\mathrm{CABG} \times 4$ & Yes/Yes & Lt ICA/53\% & No & Fig. $5 a /$ Fig. $5 b$ \\
\hline
\end{tabular}

Symp./anomaly P.O.: clinical symptom or anomaly pointed out; OP.procedure: operative procedure; Ao-bif Bypass: aortobifemoral artery bypass grafting; Clamp of Asc. Ao: clamp of ascending aorta; SVG empl.: employment of saphenous vein graft anastomosed to the ascending aorta; \%sten: percentage stenosis of carotid artery; ICA: internal carotid artery; CCA: common carotid artery; Most sten. lesion of carotid art.: most stenotic lesion of carotid artery; Clin. stage: figure of clinical stage classification of quantitative cerebral blood flow; Postop. MRI: figure of postoperative brain magnetic resonance imaging
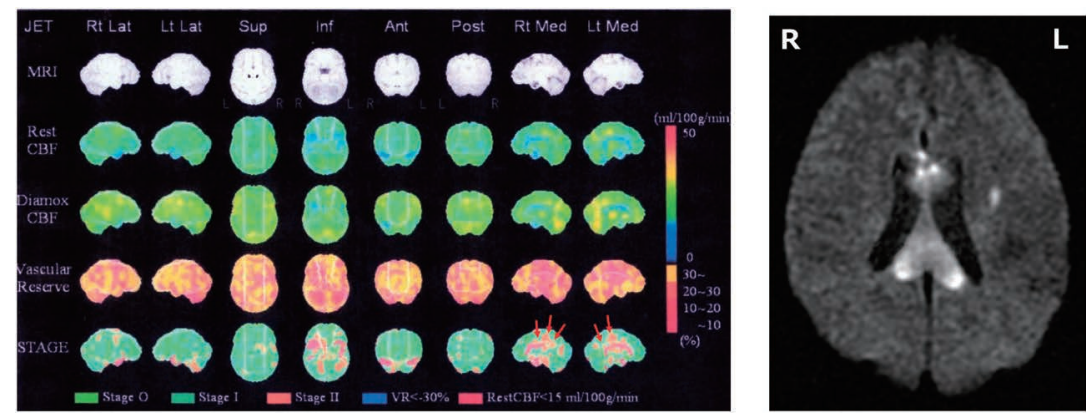

$\mathbf{a} \mid \mathbf{b}$

Fig. 1 a) Clinical stage classification of quantitative cerebral blood flow in case 1. Clinical stage II is shown for the lateral ventricle on both sides. b) Postoperative MRI in case 1. The MRI shows an acute infarction in the lateral ventricle on both sides.
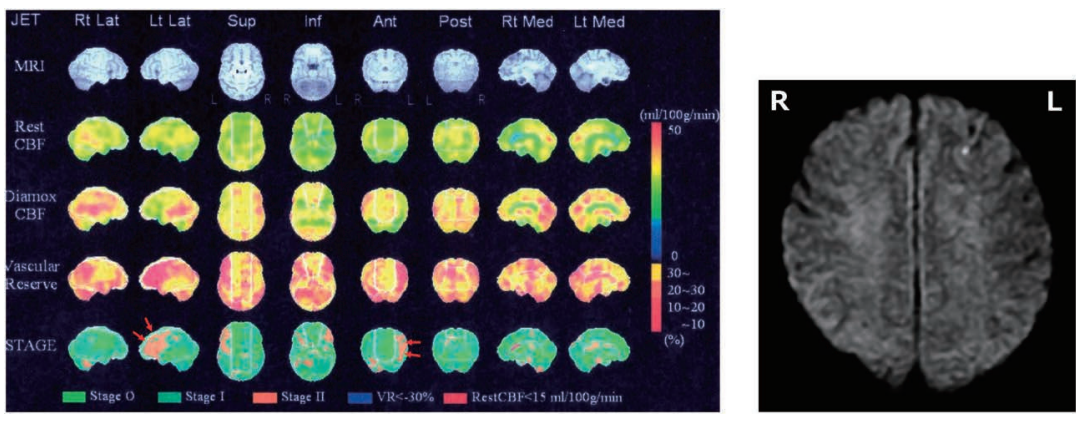

$\mathbf{a} \mid \mathbf{b}$

Fig. 2 a) Clinical stage classification of quantitative cerebral blood flow in case 2. Clinical stage II is shown in the left temporal lobe. b) Postoperative MRI in case 2. The MRI shows an acute infarction in the watershed area of the left anterior and middle cerebral artery.

\section{Discussion}

The reported incidence of stroke is $1.5 \%-3 \%$ after coronary artery bypass surgery using $\mathrm{CPB}$ and $3 \%-6 \%$ after prosthetic valve replacement. Stroke following cardiovascular surgery may decrease patient QOL, increase mortality, prolong hospital stay, and increase medical expenses, making it a primary factor in determining whether surgery is performed or not.

Nakai et al. reported that there are four possible onset mechanisms of stroke as follows ${ }^{4}$ : 1 ) a plaque-induced thrombotic mechanism such as plaque disruption, plaque erosion, and intraplaque hemorrhage; 2) a so-called embolus-induced mechanism, with emboli matter moving artery-to-artery from the major arteries within and outside of the cranium; 3) a mechanism involving low perfu- 

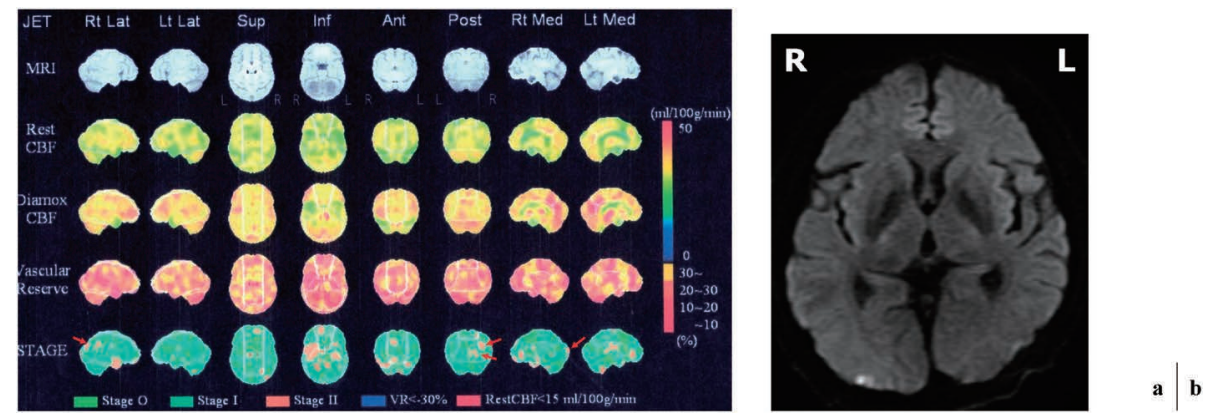

Fig. 3 a) Clinical stage classification of quantitative cerebral blood flow in case 3. Clinical stage II is shown in the right parietal and posterior lobe. b) Postoperative MRI in case 3. The MRI shows an acute infarction in the watershed area of the right parietal and posterior lobe.
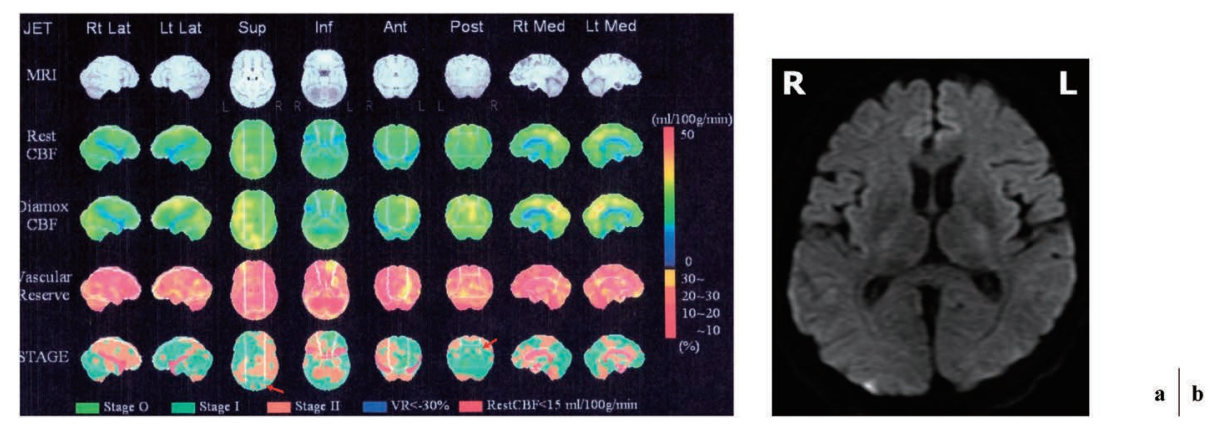

Fig. 4 a) Clinical stage classification of quantitative cerebral blood flow in case 4 . Clinical stage II is shown in the watershed area of the right middle and posterior cerebral artery. b) Postoperative MRI in case 4 . The MRI shows another acute infarction in the watershed area of the right parietal and posterior lobe.
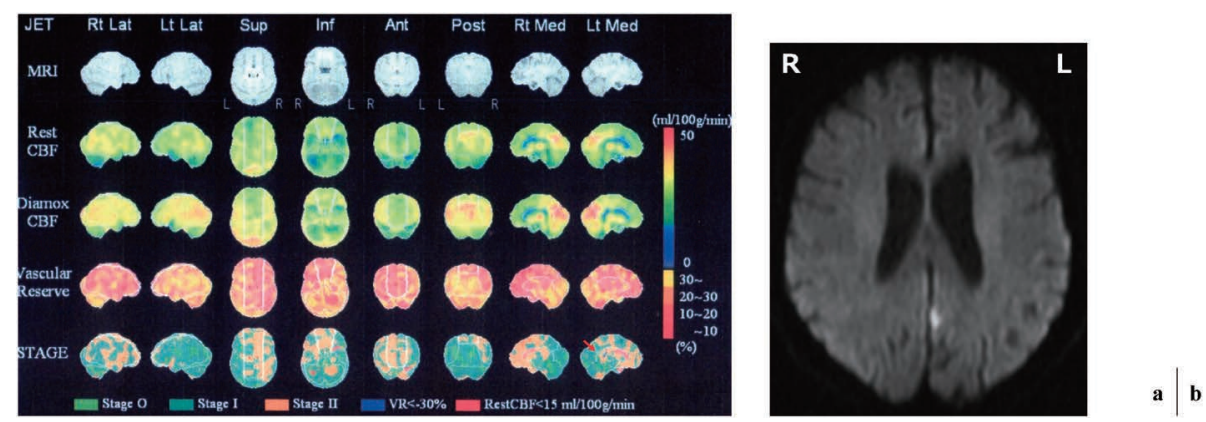

Fig. 5 a) Clinical stage classification of quantitative cerebral blood flow in case 5. Clinical stage II is shown in the posterior of the right ventricle. b) Postoperative MRI of case 5. The MRI shows an acute infarction in the in the posterior of the right ventricle.

sion pressure caused by low blood pressure and/or severe stenosis; and 4) the occlusion of penetrating branches caused by plaque growth on a major artery wall.

In the context of stroke occurring after cardiovascular surgery, and specifically considering the intraoperative progression of atherosclerosis in 4), we believe that the intraoperative growth of arterial plaque is unlikely and is thus not highly important to consider as a cause of stroke. Therefore, we believe that perioperative stroke including intraoperative stroke can be examined by applying the three factors above, excluding 4).

To avoid postoperative stroke after cardiovascular surgery caused by factors 1 )-3), we implemented countermeasures such as identifying the location of plaque in the ascending aorta, performing manipulations of the proximal anastomosis site of the bypass graft with care, and using the aorta non-touch method ${ }^{5,6}$; however, it is difficult to completely eliminate postoperative stroke.

With recent developments and popularization of cerebral blood flow measurement methods, successive reports 
have indicated that even in patients with the occlusion of the major cerebral arterial trunks, hemodynamically, patients in a state of cerebral ischemia are significantly prone to the recurrence of cerebral ischemia symptoms in the future..$^{7-12)}$

In the field of cardiovascular surgery, procedures using $\mathrm{CPB}$ or performed under cardiac arrest cause blood pressure to drop intraoperatively. Moreover, during off-pump coronary artery bypass, displacement of the heart causes cardiac output (CO) to decrease even if blood pressure is maintained using drugs, which results in a state prone to intraoperative cerebral ischemia caused by the stroke onset described in mechanism 3) by Nakai et al.

In the present study, all patients who developed stroke underwent only plain CT for preoperative close examination. Therefore, there may have been some patients in whom the lumen of the ascending aorta was characteristically poor; thus, we cannot rule out the possibility of stroke caused by embolus matter from the ascending aorta. However, even if an infarction is caused by an embolus from within the heart, the ascending aorta, or carotid artery, it is assumed that the embolus causing the infarction often arises in areas with good blood flow of stage 0 on cerebral blood flow evaluation. Regarding the possibility of stroke onset from an intra-atrial thrombus as another conceivable major cause of perioperative stroke, during the period until postoperative brain imaging, none of our patients who exhibited stroke had developed perioperative atrial fibrillation. Thus, it is unlikely that an intra-atrial thrombus developed, and even if stroke developed in this manner, it is assumed that it would occur in stage 0 areas.

Perfusion pressure, specifically cerebral hemoperfusion (HP), is produced by the heartbeat, and the pressure obtained in this manner reflects systemic blood pressure (BP). This systemic BP is primarily the pressure measured in the upper arm arteries and radial artery. Cerebral tissue perfusion is performed with this systemic BP as the pressure source; however, if there is stenosis in the arteries supplying the cerebral tissue, the stenosis can cause loss of pressure and subsequently cause the peripheral perfusion pressure to decline. The Willis arterial circle is located within the brain, and when there is no hypoplasia in this arterial circle, even if there is stenosis in the carotid artery on one side, it is possible that cerebral ischemia will not occur because the bilateral cerebral arteries are perfused by HP from the contralateral carotid artery via the Willis arterial circle. Furthermore, the Willis arterial circle includes HP from the vertebral arteries, and it is believed that whether cerebral ischemia is observed due to the presence or absence of carotid artery stenosis alone is attributed to the stenosis location and the state of blood flow via the arterial circle. Depending on the situation, we believe that cerebral ischemia can occur even with normal systemic BP in the presence of carotid artery stenosis and hypoplasia of the Willis arterial circle. The cerebral arteries other than those in the arterial circle are essentially functional end-arteries. Therefore, when there is stenosis in arteries other than those in the arterial circle, the peripheral perfusion pressure may decline. In such instances, even if systemic BP is normal, it is possible that cerebral tissue pressure will decline, and ischemia will occur due to hypoperfusion in the cerebral tissue. Thus, in this study, we used cerebral blood flow scintigraphy as the examination method to quantitatively detect the location believed to have developed hypoperfusion.

In cerebral blood flow scintigraphy, locations classified as stage II are hemodynamically evaluated as misery perfusion. Until systemic BP drops below $60 \mathrm{mmHg}$ in patients who do not usually have problems with cerebral blood flow, arterial dilatation occurs so that cerebral blood flow does not decline with the drop in systemic BP to compensate for cerebral blood flow. However, in patients with stage II, the compensatory mechanism via blood vessel dilatation does not function due to the influence of cerebral arterial stenosis caused by atherosclerosis, and at the time of preoperative cerebral blood flow scintigraphy, cerebral blood flow has already declined. In those patients, it is reported that, the oxygen uptake efficiency of brain cells has already been increased to avoid stroke onset caused by poor blood flow. ${ }^{13)}$ Thus, we believe that, in such tissue where the oxygen uptake efficiency is already increased, a further decline of blood flow during operative procedure might increase the possibility of stroke.

On the basis of the postoperative imaging results of our study, stroke only appears in stage II cases by measuring cerebral blood flow levels on cerebral blood flow scintigraphy. Moreover, it reveals that patients with stage II cerebral blood flow occupying a large area of the cortical surface are prone to stroke. Furthermore, regarding the location of stroke onset, most infarctions occurred in the watershed area where cerebral blood flow is likely the poorest. If the stroke is caused by an embolus, it is considered highly probable that the embolus flowed to a location with good blood flow on cerebral blood flow scintigraphy and that stroke occurred in this location. In the present study, we believe that it is highly probable that stroke occurred in a location different from the location of stroke onset.

Considering the timing of stroke onset, our institution attempted to maintain the systolic blood pressure (SBP) at least $\geq 80 \mathrm{mmHg}$ during off-pump bypass surgery. In the present study, although patients who exhibited infarction on postoperative imaging displayed intraoperative BP decreases to around $70 \mathrm{mmHg}$ for approximately $5-10 \mathrm{~min}$, in general, BP was maintained at $\geq 80 \mathrm{mmHg}$, and accord- 
ing to the postoperative ICU charts of cases $1-5$, SBP was generally maintained at $\geq 90 \mathrm{mmHg}$.

In all patients, the SBP at the time of low intraoperative $\mathrm{BP}$ was lower than the SBP reported in the ICU.

Although such changes in BP are considered difficult to completely avoid in off-pump bypass surgery, stroke may be caused by a further intraoperative decline in the BP of brain tissue that already had misery perfusion on preoperative normal BP testing.

During this examination of stroke, we did not conduct intraoperative measurements of brain tissue oxygen saturation $\left(\mathrm{SO}_{2}\right)$, and we could not identify the time when the brain tissue $\mathrm{SO}_{2}$ was the lowest. However, on the basis of the time when the cerebral perfusion pressure was the lowest, the stroke onset was more likely to have occurred intraoperatively than postoperatively.

In the patients in this study, the rate of stroke onset following off-pump bypass surgery based on postoperative imaging was $33 \%$. If perioperative $\mathrm{BP}$, including intraoperative BP, were to decline further, we believe that the incidence of perioperative stroke would increase and lead to asymptomatic stroke onset.

We can reach some conclusions on the basis of the present findings to help prevent perioperative stroke. Patients in whom preoperative cerebral blood flow measurements reveal that stage II cerebral blood flow occurs in $\geq 10 \%$ of the hemisphere surface area when performing coronary artery bypass, there is a high rate of postoperative stroke onset. As a countermeasure in preventing stroke, performing off-pump coronary artery bypass graft $(\mathrm{CABG})$ alone may be insufficient. Further examination of surgery performed with an increased CPB pump flow rate and maintaining a high flow rate for the CPB pump under cardiac arrest may be necessary to determine whether this protocol could lead to the prevention of intraoperative stroke.

Lastly, for the cerebral blood flow levels measured by cerebral blood flow scintigraphy in the present study, five days were necessary for the tests with interval three days between the two scintigraphy scans. Furthermore, because radioisotopes are used, the test requires prior planning, which is not easy for all subjects. Therefore, we were forced to narrow down the number of study subjects, without overlooking postoperative stroke as much as possible. The subject sample was limited to those with carotid artery stenosis of $50 \%$ and who provided consent. Thus, the present study only included 33 patients.

In the present study, the proportion of $10 \%$ of the cerebral hemisphere area displaying stage II cerebral blood flow was defined as the criteria for predicting the onset of postoperative stroke. For patients who exhibited preoperative lacunar infarction only, postoperative imaging revealed no onset of stroke, and the mentioned preoperative lacunar infarction was inferred to likely not be a major risk factor of the risk of postoperative stroke onset. In this respect, further examination is warranted; however, anatomically, when infarction has already developed in the small branches that causes lacunar infarction, it was thought to be a possibility because there is little perfusion in the more peripheral region. Therefore, to exclude this possibility, we wanted to exclude small areas of ischemia in the present study; thus, we excluded patients with stage II cerebral blood flow in $<10 \%$ of cerebral hemisphere areas from our analysis of the risk of postoperative stroke onset. Consequently, we found that this measurement was useful for the prediction of stroke with a statistically significant difference.

However, at this point in time, it is impossible to determine which percentage of the stage II area can predict postoperative stroke with the most accuracy due to insufficient subject sample size. We believe that a future prospective study is necessary.

\section{Conclusion}

Stroke following cardiovascular surgery may be caused by an embolus or by low preoperative cerebral blood flow reserve, with low intraoperative $\mathrm{BP}$ and $\mathrm{CO}$.

The onset of postoperative stroke caused by low cerebral blood flow reserve is considered difficult to determine by the degree of carotid artery stenosis on preoperative examination alone.

The results of the present study reveal that, in patients with $\geq 10 \%$ of the hemisphere area displaying stage II cerebral blood flow as measured using the stereotactic extraction estimation (SEE)-JET study method, the rate of postoperative stroke is frequent with a statistically significant difference. Thus, we believe that the cerebral blood flow measurement method used in the present study is useful for predicting the onset of stroke following cardiovascular surgery.

\section{Disclosure Statement}

There are no conflicts of interest in the present paper.

\section{References}

1) JET Study Group. Japanese EC-IC Bypass Trial (JET Study): study design and interim analysis. Surgery for Cerebral Stroke 2002; 30: 97-100. (in Japanese)

2) Nakagawara J. Clinical neuroimaging of cerebral ischemia. Brain and Nerve 1999; 51: 502-13. (in Japanese)

3) Kanda Y. Investigation of the freely available easy-touse software "EZR" for medical statistics. Bone Marrow Transplant 2013; 48: 452-8.

4) Nakai K, Nagata H, Fujii K, et al. A therapeutic usage of cilostazol for a patient with symptomatic intracranial arte- 
rial stenosis. Progress in Medicine 2010; 30: 1773-6. (in Japanese)

5) Bergman P, Hadjinikolaou L, Dellgren G, et al. A policy to reduce stroke in patients with extensive atherosclerosis of the ascending aorta undergoing coronary surgery. Interact Cardiovasc Thorac Surg 2004; 3: 28-32.

6) Hirose H, Amano A, Takahashi A, et al. Off-pump total arterial bypass grafting using bilateral mammary, gastroepiploic, and radial artery. Ann Thorac Cardiovasc Surg 2000; 6: 405 7.

7) Derdeyn CP, Grubb RL Jr, Powers WJ. Cerebral hemodynamic impairment: methods of measurement and association with stroke risk. Neurology 1999; 53: 251-9.

8) Grubb RL Jr, Derdeyn CP, Fritsch SM, et al. Importance of hemodynamic factors in the prognosis of symptomatic carotid occlusion. JAMA 1998; 280: 1055-60.

9) Kuroda S, Houkin K, Kamiyama H, et al. Long-term prognosis of medically treated patients with internal carotid or middle cerebral artery occlusion: can acetazolamide test predict it? Stroke 2001; 32: 2110-6.

10) Webster MW, Makaroun MS, Steed DL, et al. Compromised cerebral blood flow reactivity is a predictor of stroke in patients with symptomatic carotid artery occlusive disease. J Vasc Surg 1995; 21: 338-44; discussion, 344-5.

11) Widder B, Kleiser B, Krapf H. Course of cerebrovascular reactivity in patients with carotid artery occlusions. Stroke 1994; 25: 1963-7.

12) Yamauchi H, Fukuyama $H$, Nagahama $Y$, et al. Evidence of misery perfusion and risk for recurrent stroke in major cerebral arterial occlusive diseases from PET. J Neurol Neurosurg Psychiatry 1996; 61: 18-25.

13) Matsumoto Y, Ogasawara K, Saito H, et al. Detection of misery perfusion in the cerebral hemisphere with chronic unilateral major cerebral artery steno-occlusive disease using crossed cerebellar hypoperfusion: comparison of brain SPECT and PET imaging. Eur J Nucl Med Mol Imaging 2013; 40: 1573-81. 